\title{
e-Migrinter
}

$18 \mid 2019$

L'ethnographie en migration(s)

\section{Pause Pédagogique au Sénégal}

David Lessault et Tehem

\section{(2) OpenEdition}

Journals

Édition électronique

URL : https://journals.openedition.org/e-migrinter/1519

DOI : 10.4000/e-migrinter.1519

ISSN : 1961-9685

Éditeur

UMR 7301 - Migrinter

Référence électronique

David Lessault et Tehem, «Pause Pédagogique au Sénégal », e-Migrinter [En ligne], 18 | 2019, mis en ligne le 01 mai 2019, consulté le 20 mai 2021. URL : http://journals.openedition.org/e-migrinter/1519 ; DOI : https://doi.org/10.4000/e-migrinter.1519

Tous droits réservés 


\section{Pause pédagogique Au Sénégal}

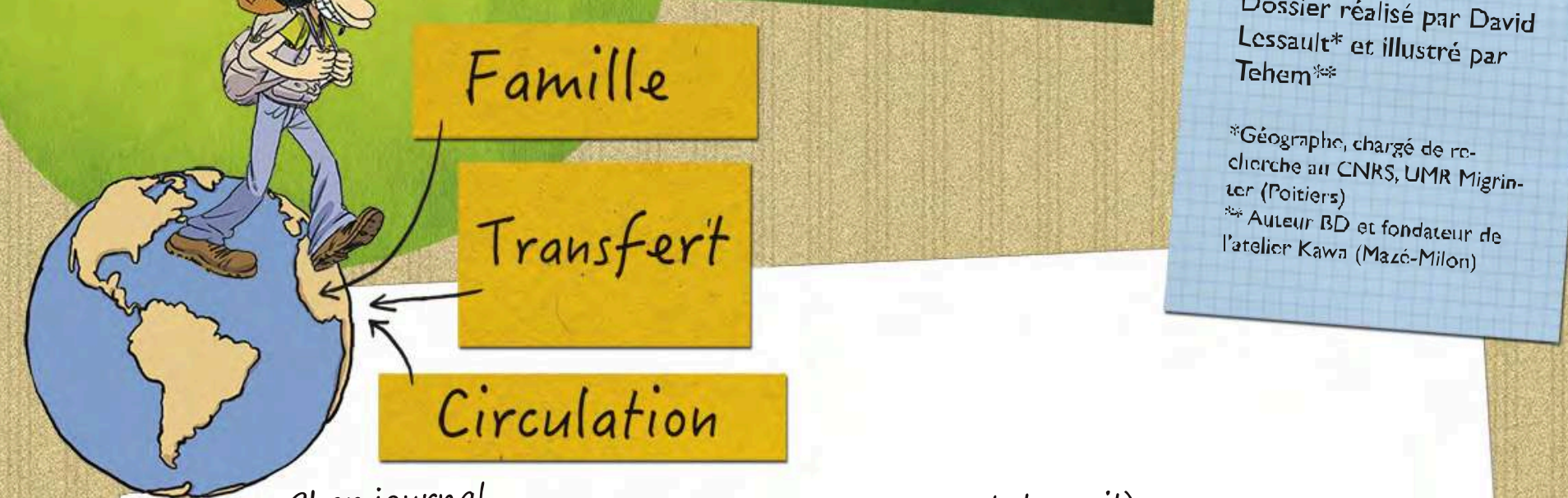

Auteurs

Dossier réalisé par David Lessault* et illustré par

"Géographe, chargé de reclecrche au CNRS, UMR Migrin(Poitiers)

* Auleur BD es fondateur de

Cher journal,

Je suis arrivé avant-hier à Dakar $6: 30$ (vol de nuit). 12 :00 (Premières impressions après lecture et brefs échanges avec les gens du coin).

Dakar est la capitale du Sénégal, un petit pays situé en Afrique de l'Ouest. Il borde la côte Atlantique mais à l'intérieur le climat est plus chaud et sec, on entre dans la zone aride du Sahel. Je comprends mieux pourquoi la majorité des habitants sont installés dans les villes côtières. En raison de plusieurs épisodes de sécheresse dans les années 1970, la pratique des cultures et de l'élevage est devenue difficile et l'activité peu rentable. De nombreux paysans ont alors migré vers les grandes villes pour chercher un emploi et aider leur famille à mieux vivre dans les villages de l'intérieur s'ils hamille au village et leur apporte ler, ils reviennentier qui leur procure souvent une grande estime des parents, femmes ou enfants restés sur place. Ici on est solidaire!

Depuis quelques années, la crise économique s'est généralisée dans les villes autrefois dynamiques. A Dakar, qui compte aujourd'hui plus de trois millions d'habitants (dont plus de la moitié a moins de 20 ans) les jeunes peinent à trouver du travail, même lorsqu'ils ont des diplômes ou une formation. L'Etat du Sénégal ne dispose pas de suffisamment de moyens pour intégrer nombreuses pour offrir un emploi à et les entreprise chômage reste extrêmement élevé et beaucoup tous. Le taux eux désespèrent.

On m'a invité à boire le thé, hier soir (l'hospitalité sénégalaise - la Terranga - n'est pas un mythe). Je n'ai entendu parler que d'Europe, de partir quoi qu'il en coûte, à pieds ou en pirogue pour tenter sa chance ailleurs «|ci il n'y a rien ». Certains semblent frustrés, envieux des « expat » qui marient les plus belles femmes et ont de grandes demeures à construire pour leurs parents parce qu'ils ont réussi en France... Les « vieux 》 (qualificatif courant donné aux anciens: marque le respect) les découragent, le voyage est selon eux risqué et l'Europe n'est pas toujours l'eldorado qu'on présente...

Qu'en penser? 
En réalité, l'émigration ou plutôt la mobilité internationale des Sénégalais, est une pratique très ancienne et répandue en Afrique de l'Ouest. De nombreuses études menées depuis plus de 40 ans par des chercheurs en sciences sociales ont montré comment la migration de certains membres permettait d'aller chercher ailleurs, parfois dans d'autres pays, de nouvelles ressources économiques pour permettre à la famille de subvenir à ses besoins en toutes circonstances (faibles récoltes, aléas climatiques, etc.). Pour les jeunes hommes, aller travailler en ville le temps de "l'hivernage" (saison des pluies), se faire embaucher sur un chantier d'un pays côtier pour quelques années, ou commercer en itinérant à l'étranger constituent une pratique courante qui a essaimé les Sénégalais dans l'ensemble des pays d'Afrique de l'Ouest.

L'émigration sénégalaise vers l'Europe est un phénomène plus récent et de moindre importance, bien qu'il soit régulièrement mis en scène de façon spectaculaire et dramatique dans les médias (naufrages de pirogues au large des côtes marocaines ; prises d'assaut répétées des barrières de Ceuta et Melilla, etc.). En Europe, les Sénégalais se sont d'abord tournés vers la France, l'ancienne métropole duran la période coloniale. Les entrepreneurs français sont venus recruter la main d'œuvre dont ils avaient besoin jusqu'au Sénégal, afin d'assurer le développement économique, industriel, des Trente Glorieuses (1945-1975). Certains immigrés ont fait venir leur famille en France (épouse et enfants), d'autres sont restés seuls, hébergés dans des foyers de travailleurs. Avec l'arrêt officiel des migrations économiques, la politique française menée à l'époque a souvent conduit au regroupement des familles dans notre pays. Cette période marque la féminisation des migrations sénégalaises vers la France au cours des années 1980 et 1990.

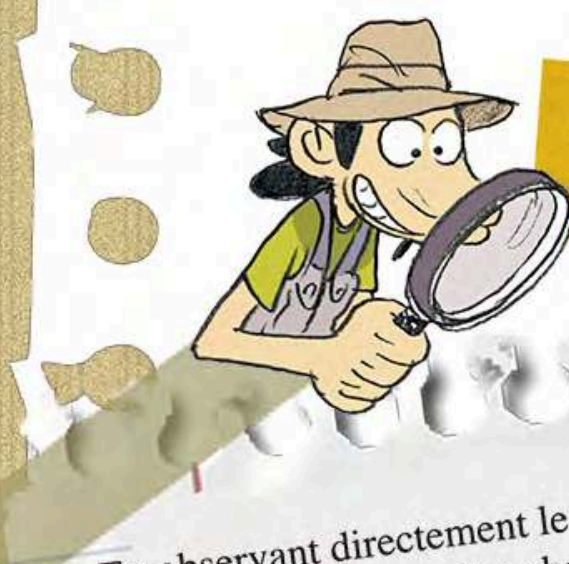

En observant directement le fonctionnement de
groupes de migrants, les chercheurs de Migrinter ont élaboré la notion de « circulation migratoire » fondée sur la mise en évidence de ces relations humaines, so ciales et financières à distance. Certains d'atistiques qui peront contribué à produire des statistiquements, de mieux mettent de mesurer ces mouvem la nature des échanges entre connaître les parcours émigration. Les enquêtes le Sénégal et les pays migrants et de leur famille, au conduites auprès des migr pays européens permettent Sénégal et dans plusieurs pannées statistiques qui aujourd'hui de disposer de cette vision de l'émigra-

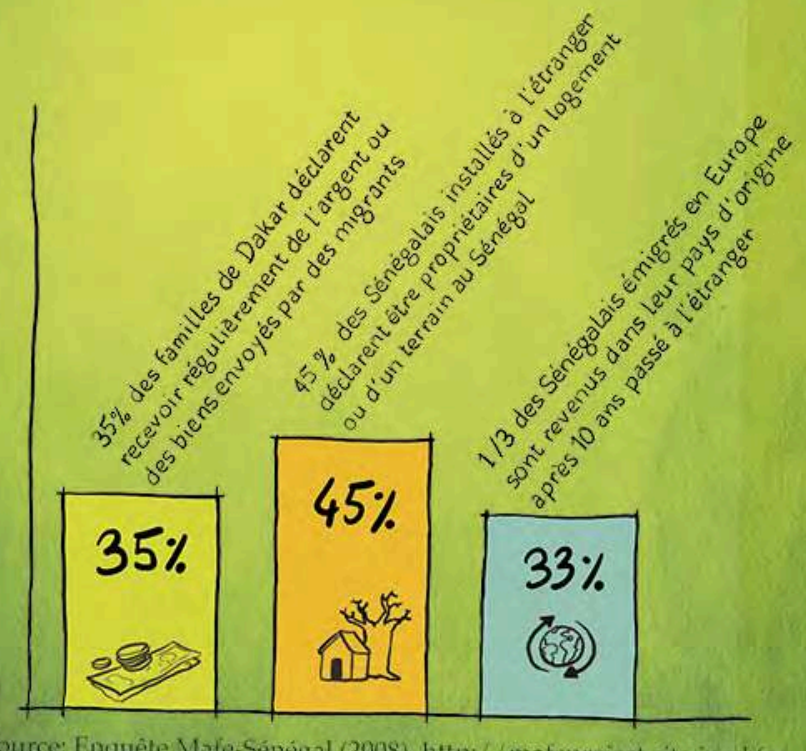
confortent la pertinence de cette tion en termes de circulation. 


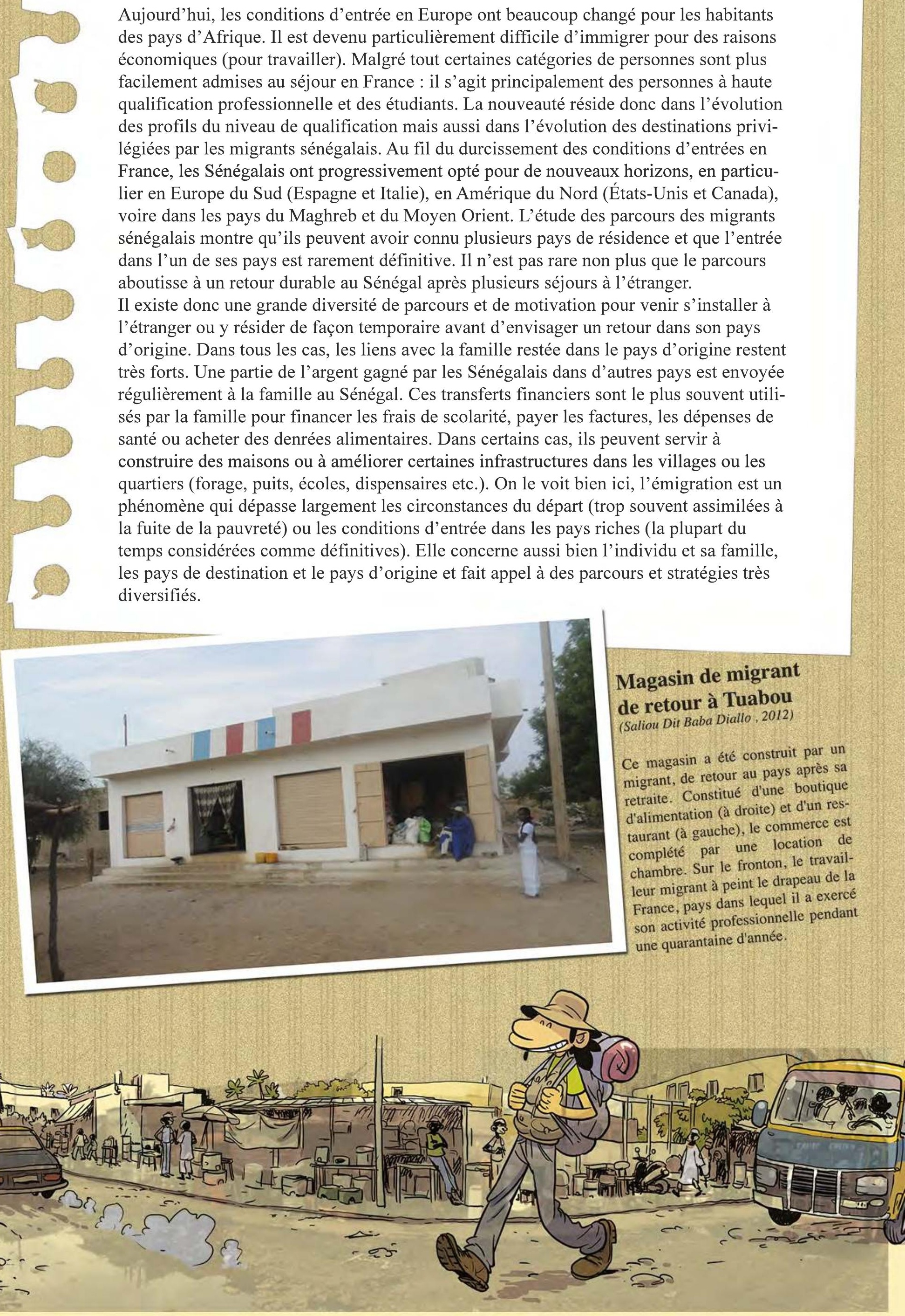


UN PIED AU PARADIS
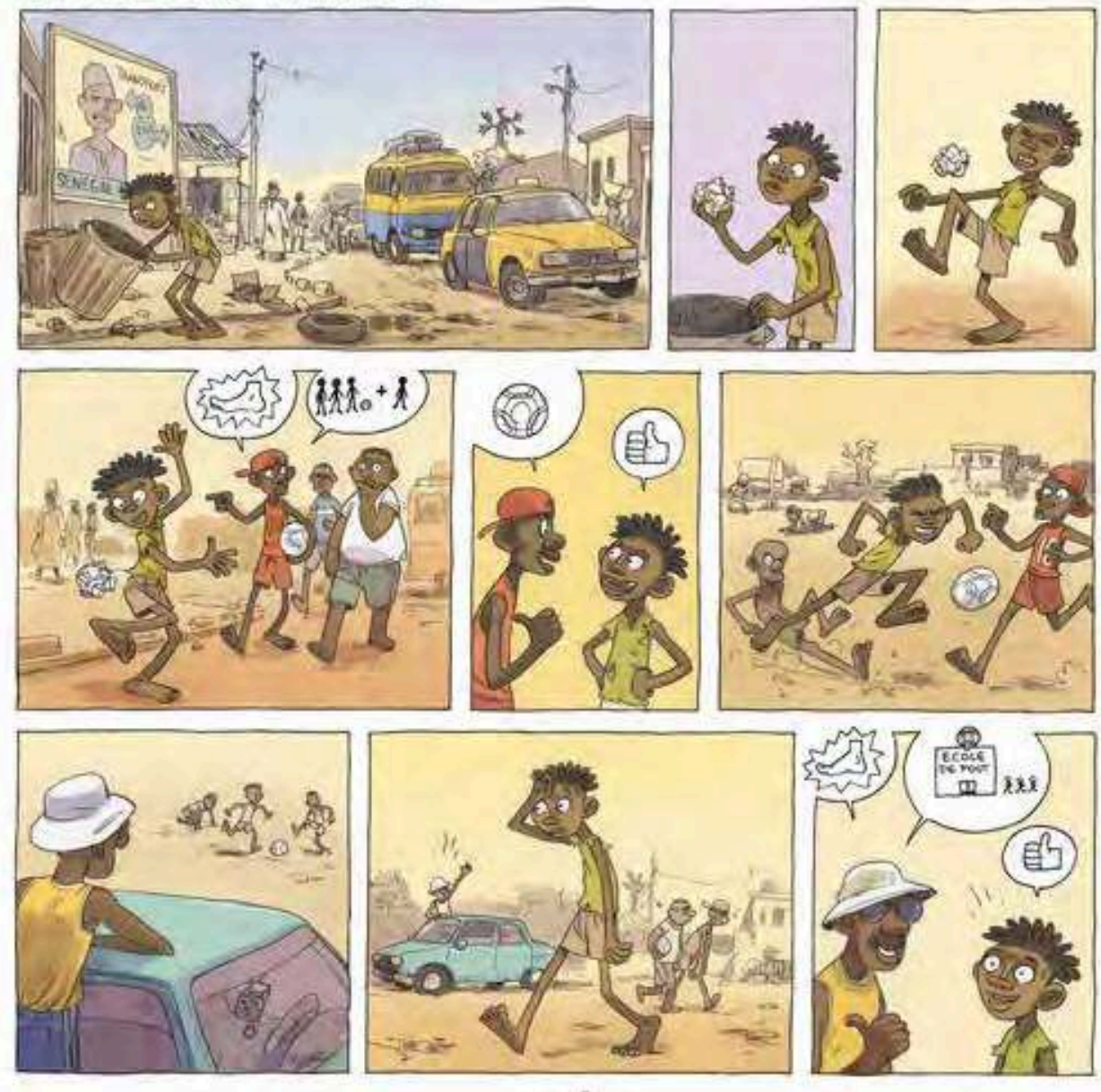
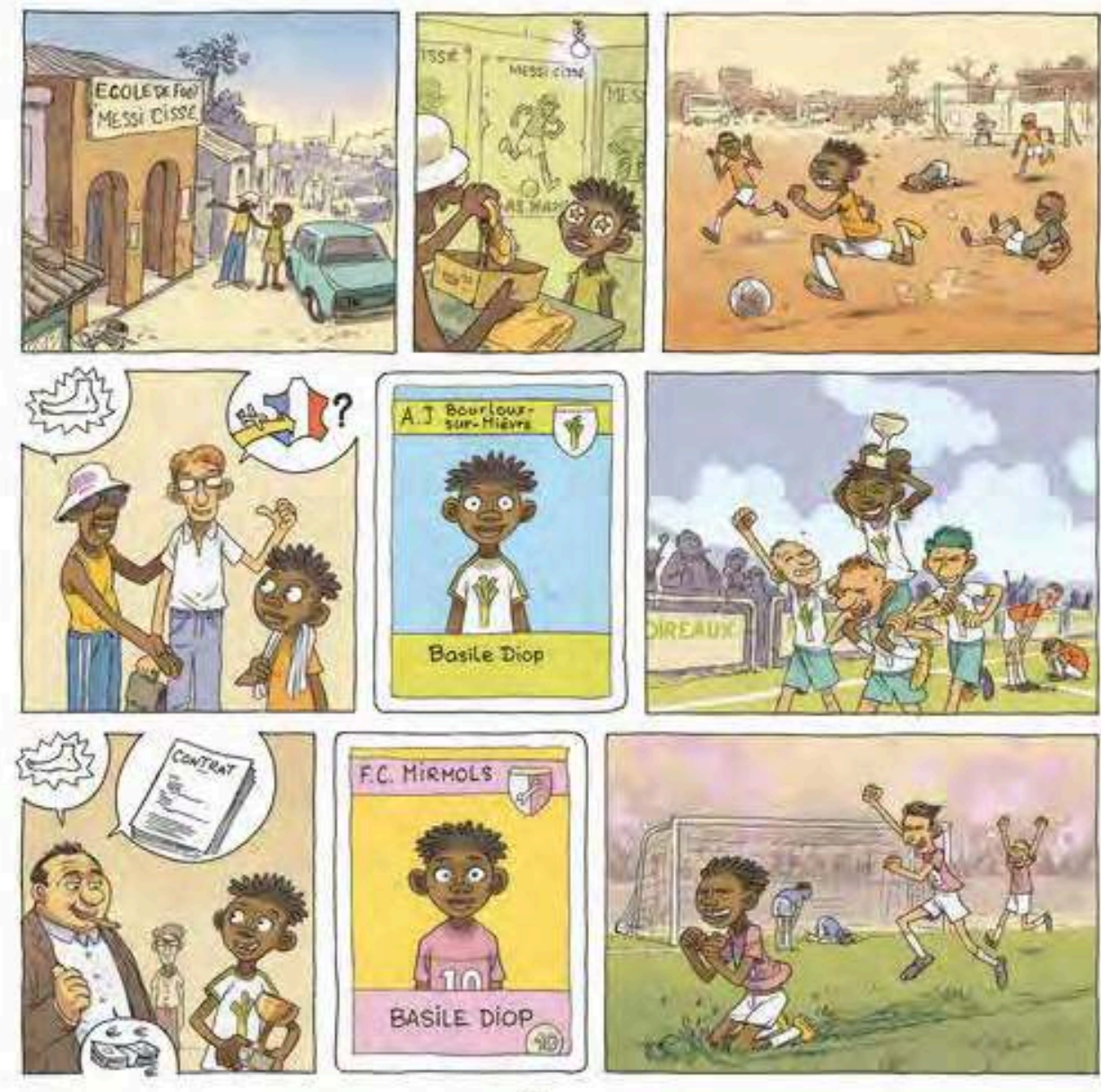

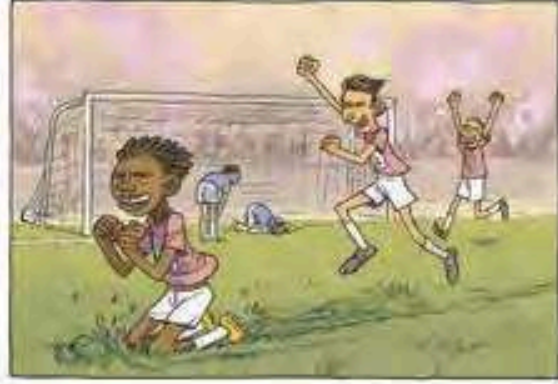



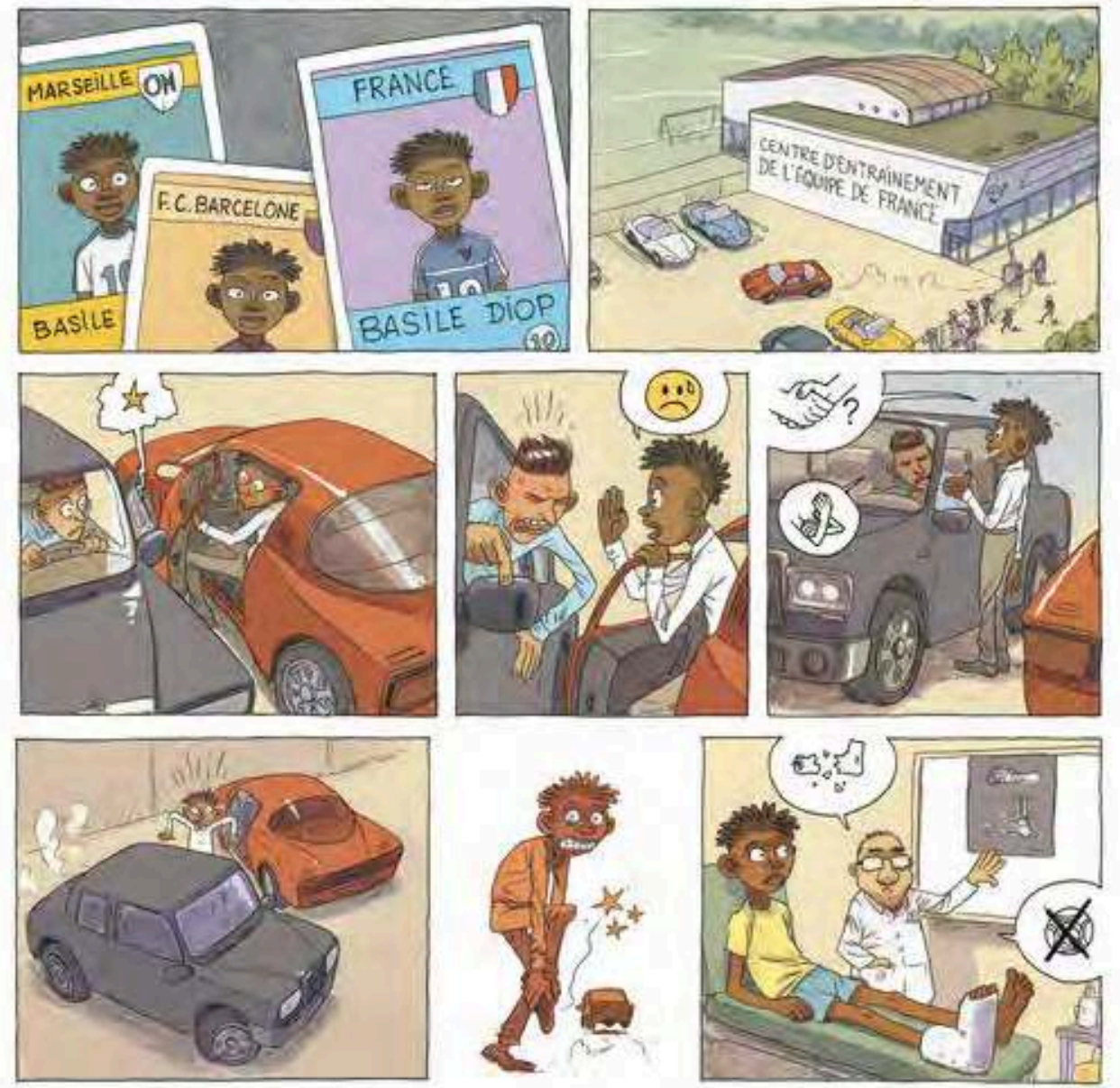

3
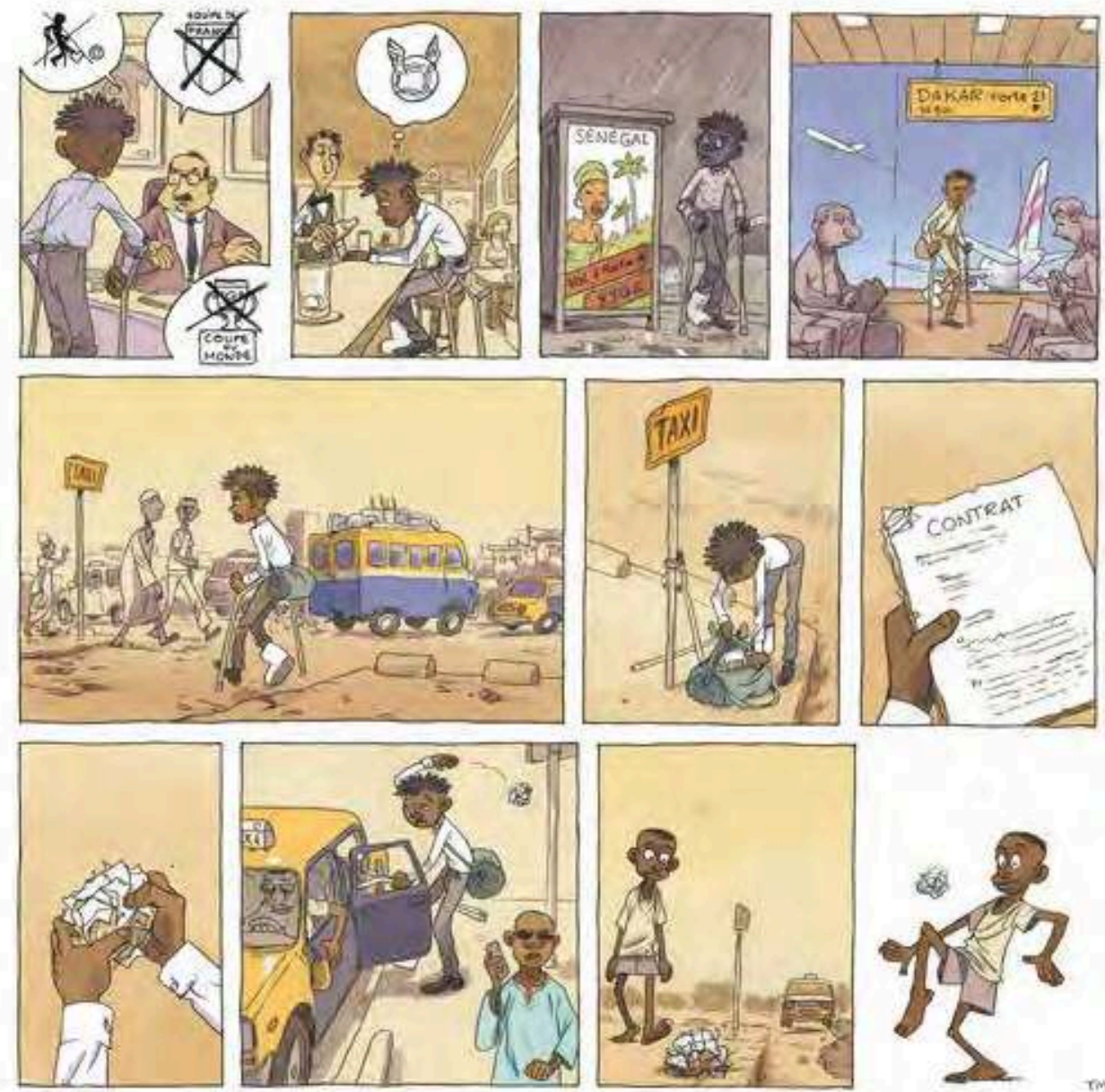


\section{Je retiens...}

Famille : la succession des mouvements migratoires conduit à la dispersion des membres d'une même famille dans plusieurs lieux. Quand cette dispersion se réalise à l'échelle internationale, on parle de plus en plus de « famille transnationale », dont l'organisation du moment est structurée au-delà les frontières. Plus qu'une décision individuelle, la migration internationale est souvent une stratégie qui s'élabore au niveau du groupe familial et engage des attentes de la part des membres de la parenţé qui ne migrent pas.

Transfert : caractérise généralement l'ensemble des dons, en argent ou en nature, adressés par les migrants internationaux à leur famille restée dans le pays d'origine. Quand la migration internationale atteint un niveau important, les transferts deviennent un enjeu économique fort pour améliorer les conditions de vie et soutenir le développement du pays, notamment dans les pays pauvres.

Circulation : manière de penser la migration internationale qui correspond mieux à la dynamique des déplacements humains observée dans les recherches en sciences sociales. Elle permet de ne plus réfléchir à la migration uniquement comme un mouvement d'entrée définitive dans les pays d'accueil mais comme un phénomène réversible (migration de retour) et comme un ensemble de relations humaines et économiques entre les pays concernés (pays d'accueil, pays d'origine, autres pays d'installation). La gestion des migrations internationales devient alors un phénomène global et une préoccupation partagée par l'ensemble des états.

\section{J'approfondis...}

Lessault, David ; Robin, Nelly (2015) Notice « Sénégal », in Simon, G. (coord.) Dictionnaire des migrations internationales. Approche géohistorique, Paris, Armand Colin, pp. 471-475. Beauchemin, Chis ; Lessault, David (2014) Les statistiques des migrations africaines : ni invasion, ni exode, e-Migrinter, $\mathrm{n}^{\circ} 12$, pp. 32-43. [Disponible sur Internet].

Lessault, David ; Beauchemin, Cris (2009) Les migrations d'Afrique subsaharienne vers l'Europe : un essor encore limité, Population et Sociétés, $n^{\circ}$ 452, 4 p. [Disponible sur Internet]. Bon, la migration internationale est un phénomène social et historique important an Sénégal... C. est visiblement une donnée économique à prendre en compte dans la politique de développement du pays. Pour autant, elle concerne essentiellement aujourd'hui les familles les mieux dotées qui disposent de ressources suffisantes pour financer le voyage en Europe, accéder aux emplois qualifiés et assurer l'installation dans des pays où le coût de la vie est bien plus élevé qu' au Sénégal. Si cela fait rêver les jeunes issus des familles les plus modestes c'est certainement parce que I'horizon sur place leur semble bien sombre... Heureusement, peu d'entre eux tentent de partir par des voies illégales et bien souvent risquées.

\section{J'illustre...}

Lien vers le film Sembène : https://whw Mandat (1968) 》 de Ousmane =TYCbRyr7IS4.
.

: http://www aller du film « La pirogue (2012) dia $=19400338 \&$ cfilm $=206433$ /video/player_gen cmeLien vers le pdf tine, l'aventure de la BD « Émigration clandesmortelle (2006)

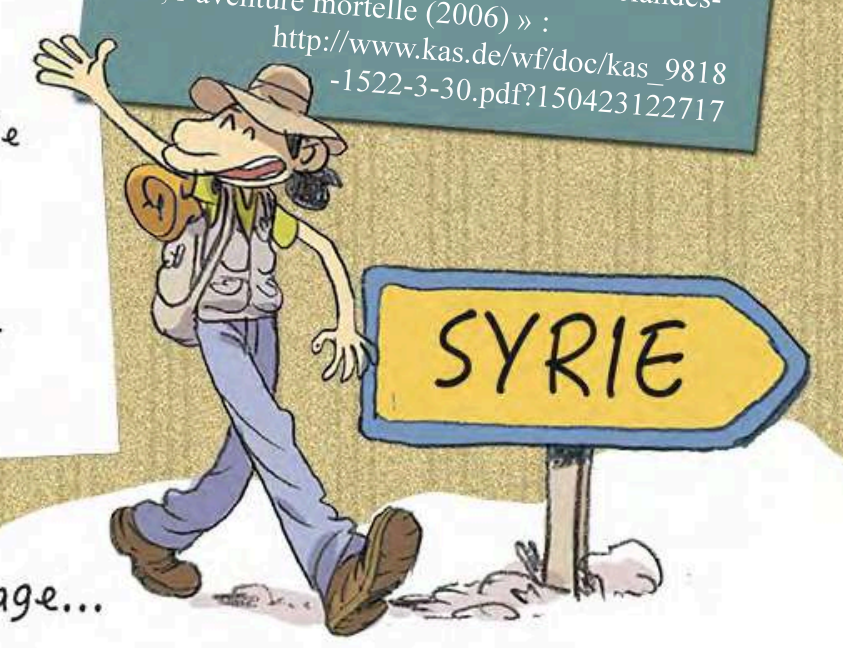

